\title{
Supervivencia de pacientes pediátricos con inmunodeficiencias primarias en un hospital público del occidente de México
}

\author{
Survival of pediatric patients with primary immunodeficiencies in a public \\ hospital in western Mexico
}

\author{
Dra. María E. Núñez-Núñeza, Dr. Juan C. Lona-Reyes b,c, Dra. Sandra I. Cortés-González ${ }^{d, e}$, \\ Dra. Regina Mallinalli Navarro-Martín del Campof, Dra. Ana L. Orozco-Alvaradof, \\ Dr. Oscar González-Ramellaf y Dr. Luis Gustavo Orozco-Alatorred,f
}

\section{RESUMEN}

Se presenta una serie de casos deinmunodeficiencias primariasy se describen las variables asociadas a supervivencia en pacientes $\leq 16$ años. Los diagnósticos fueron acordes a los criterios de la Unión Internacional de las Sociedades de Inmunología. Se realizó un análisis de supervivencia mediante curvas de Kaplan-Meier.

Entre los años 2004 y 2019, se diagnosticaron 40 pacientes con inmunodeficiencias primarias. Las más frecuentes fueron inmunodeficiencias que afectaban la inmunidad celular y humoral, el 32,5\%, y deficiencias predominantemente de anticuerpos, el $32,5 \%$. La mediana de edad al inicio de los síntomas y al momento del diagnóstico fue de 3,01 y 10,4 meses, respectivamente. Fallecieron el $35 \%$ y el riesgo fue mayor en pacientes con inmunodeficiencias que afectaban la inmunidad celular y humoral y en quienes presentaron manifestaciones clínicas y tuvieron el diagnóstico en los primeros seis meses de vida.

Palabras clave: enfermedades de inmunodeficiencia primaria, inmunodeficiencia combinada grave, deficiencia de anticuerpos primaria, sistema inmunológico, enfermedades del sistema inmune.

http: / / dx.doi.org/10.5546/aap.2021.202

Texto completo en inglés:

http:/ / dx.doi.org/10.5546/ aap.2021.eng.202

a. Hospital Civil de Guadalajara "Dr. Juan I. Menchaca", División de Pediatría, Servicio de Alergia e Inmunología Clínica Pediátrica. Guadalajara, Jalisco, México.

b. Hospital Civil de Guadalajara "Dr. Juan I. Menchaca”, División de Pediatría, Servicio de Infectología, Guadalajara, Jalisco, México.

c. Universidad de Guadalajara, Centro Universitario de Tonalá, Tonalá, Jalisco, México.

d. Hospital Civil de Guadalajara "Dr. Juan I. Menchaca", División de Pediatría, Guadalajara, Jalisco, México.

e. Universidad de Guadalajara, Centro Universitario de Ciencias de la Salud, Guadalajara, Jalisco, México.

f. Hospital Civil de Guadalajara "Dr. Juan I. Menchaca”, División de Pediatría, Unidad de Trasplante de Médula Ósea, Guadalajara, Jalisco, México.

Correspondencia:

Dr. Juan C. Lona Reyes: carloslona5@hotmail.com

Financiamiento: Ninguno.

Conflicto de intereses: Ninguno que declarar.

Recibido: 18-9-2020

Aceptado: 29-10-2020
Cómo citar: Núñez-Núñez ME, Lona-Reyes JC, Cortés-González SI, Mallinalli Navarro-Martín del Campo R, et al. Supervivencia de pacientes pediátricos con inmunodeficiencias primarias en un hospital público del occidente de México. Arch Argent Pediatr 2021;119(3):202207.

\section{INTRODUCCIÓN}

Las inmunodeficiencias primarias (IDP) son defectos inherentes de la inmunidad innata o adaptativa que se manifiestan con infecciones frecuentes y/o graves, síntomas de autoinmunidad, linfoproliferación, alergia y eventos oncológicos. ${ }^{1-4}$ Se desconoce la prevalencia mundial de las IDP. En América Latina, se ha estimado una prevalencia de un evento / 516000 habitantes, pero es probable que exista un subdiagnóstico. ${ }^{5}$ En México, en las últimas décadas, se ha triplicado el número de casos reportados. ${ }^{6}$

A pesar de la información disponible sobre IDP, es frecuente un diagnóstico tardío. El objetivo de este estudio fue describir una serie de casos de IDP en pacientes pediátricos y las variables asociadas a la supervivencia.

\section{MATERIAL Y MÉTODOS}

Se realizó un estudio observacional retrospectivo, en el Hospital Civil de Guadalajara "Dr. Juan I. Menchaca" (HCGJIM) de la ciudad de Guadalajara, Jalisco, México. La institución brinda servicios de salud a población abierta de escasos recursos económicos.

Se incluyeron en el estudio los pacientes $\leq 16$ años a quienes se les hubiera diagnosticado IDP en la Institución del 1 de enero de 2004 al 31 de diciembre de 2019. No se incluyeron los pacientes con inmunodeficiencias secundarias.

Los diagnósticos y clasificación de las IDP fueron acordes a los criterios de la Unión Internacional de las Sociedades de Inmunología.7,8 La información sobre las variables se obtuvo de los expedientes clínicos (edad de inicio de 
los síntomas y al momento del diagnóstico, antecedentes familiares y de hospitalizaciones, manifestaciones clínicas, género, tipo de IDP, tratamiento y defunciones).

Se definió como "tiempo de retraso en el diagnóstico" el transcurrido entre el inicio de los síntomas y el diagnóstico, y "tiempo de supervivencia" el trascurrido desde el diagnóstico de IDP hasta la muerte o hasta el momento de registro de información para este estudio en los pacientes vivos. Las muertes tempranas fueron aquellas que se presentaron en el primer año de vida. Se consideraron los padres como consanguíneos si presentaron parentesco biológico de primos segundos o más próximos.

\section{Análisis estadístico}

De las variables cualitativas, se estimaron frecuencias y porcentajes, y de las variables cuantitativas, mediana, mínimo y máximo. Para el contraste de hipótesis, se usó la prueba $\chi^{2}$ para comparar proporciones y U de Mann-Whitney para comparar medianas. Se efectuó el análisis de supervivencia mediante las curvas de KaplanMeier con prueba log rank para el contraste de hipótesis. Se utilizó el programa IBM SPSS Statistics Versión 20. El proyecto fue aprobado por los Comités de Ética e Investigación del HCGJIM, con registro 0372/ 20 HCGJIM2020.

TABLA 1. Inmunodeficiencias primarias diagnosticadas durante el período de estudio

\begin{tabular}{|c|c|c|}
\hline & $\mathrm{n}, \%$ & Método diagnóstico \\
\hline $\begin{array}{l}\text { Inmunodeficiencias que afectan } \\
\text { la inmunidad celular y humoral }\end{array}$ & $13(32,5)$ & \\
\hline Inmunodeficiencia combinada grave & 10 & $\begin{array}{l}\text { Linfopenia grave, (T-, } \mathrm{B}+/-\mathrm{y} \mathrm{NK}+/-) \text {. Inmunoglobulinas bajas, } \\
\text { asociadas con infecciones oportunistas. } \\
\text { Defecto genético corroborado en tres (RAG1, RAG2, e IL2RG) }\end{array}$ \\
\hline Síndrome de hiper-IgM & 2 & $\begin{array}{l}\text { Linfocitos T+, B+, IgM elevada, IgG, IgA, IgE menores que los } \\
\text { valores normales para la edad. Infecciones por patógenos } \\
\text { oportunistas (tuberculosis miliar y colangitis). Uno con deficiencia } \\
\text { de CD } 40 \mathrm{~L} \text { y otro con deficiencia de CD } 40 \text {. }\end{array}$ \\
\hline Deficiencia de CD4 & 1 & $\begin{array}{l}\text { Linfocitos T (CD3) menores que los valores normales, a expensas } \\
\text { de linfocitos T CD4 (bajos). Linfocitos B y NK normales. } \\
\text { Inmunoglobulinas séricas normales. Infecciones oportunistas } \\
\text { (adenitis supurativa crónica por vacuna BCG y candidiasis recurrente). }\end{array}$ \\
\hline
\end{tabular}

\begin{tabular}{|c|c|c|}
\hline \multirow{2}{*}{$\begin{array}{l}\text { Deficiencias predominantemente } \\
\text { de anticuerpos } \\
\text { Agammaglobulinemia ligada al X }\end{array}$} & \multicolumn{2}{|l|}{$13(32,5)$} \\
\hline & 11 & $\begin{array}{l}\text { Linfocitos B muy bajos o ausentes. Inmunoglobulinas séricas bajas. } \\
\text { Infecciones bacterianas recurrentes (neumonía, otitis, sinusitis, } \\
\text { artritis séptica). }\end{array}$ \\
\hline Inmunodeficiencia común variable & 2 & $\begin{array}{l}\text { Hipogammaglobulinemia, infecciones recurrentes y } \\
\text { autoinmunidad }(1 / 2) \text {. }\end{array}$ \\
\hline $\begin{array}{l}\text { Inmunodeficiencias combinadas } \\
\text { con características sindrómicas }\end{array}$ & $8(20)$ & \\
\hline Síndrome de Wiskott-Aldrich & 7 & $\begin{array}{l}\text { Trombocitopenia, eccema, peso bajo, diarrea crónica. } \\
\text { No expresión WASp (3/7); el resto por asociación de familiar afectado. }\end{array}$ \\
\hline Ataxia-telangiectasia & 1 & Ataxia, telangiectasias oculares, neumonía con abscesos pulmonares. \\
\hline $\begin{array}{l}\text { Defectos congénitos del número o } \\
\text { función de fagocitos }\end{array}$ & $5(12,5)$ & \\
\hline Neutropenia congénita grave & 3 & $\begin{array}{l}\text { Abscesos cutáneos, infecciones graves recurrentes. Neutropenia } \\
\text { profunda }(C A N<100) \text {. Mutación de ELANE }(1 / 3) \text {. }\end{array}$ \\
\hline Glucogenosis & 2 & Neutropenia, hipoglucemia, hepatomegalia, hiperlipidemia. \\
\hline Defectos en la inmunidad intrínseca e innata & $1(2,5)$ & \\
\hline $\begin{array}{l}\text { Susceptibilidad mendeliana para infecciones } \\
\text { por micobacterias }\end{array}$ & 1 & $\begin{array}{l}\text { Adenitis supurativa crónica progresiva por Mycobacterium bovis y } \\
\text { Mycobacterium abscessus. Mutación IL-12R } \beta 1 \text {. }\end{array}$ \\
\hline Total & 40 & \\
\hline
\end{tabular}

RAG: gen activador de la recombinación; IL2RG: receptor gamma de interleucina 2' CD40L: ligando CD40;

BCG: bacillus de Calmette y Guérin; WASp: proteína del síndrome de Wiskott-Aldrich; CAN: conteo absoluto de neutrófilos;

ELANE: Elastase neutrophil expressed; IL-12R $\beta 1$ : receptor de interleuquina 12, sub unidad beta 1. 


\section{RESULTADOS}

Durante el período de estudio, se diagnosticaron IDP en 40 pacientes con mediana de edad al momento del diagnóstico de 10,4 meses (máximo de 194,6, mínimo de 1). El 75 \% (n: 30) fueron de género masculino. Las IDP identificadas se muestran en la Tabla 1.

Presentaron antecedente familiar de IDP 20/40; muertes tempranas en familiares de primer grado, 21/40, y padres consanguíneos en 3 casos. Al momento del diagnóstico, mostraron desnutrición el $70 \%$ (n: 28), enfermedades atópicas el 32,5\% (n: 13), y tres presentaron datos clínicos de autoinmunidad (anemia hemolítica autoinmune en 2 y trombocitopenia autoinmune en 1). Durante el seguimiento, dos tuvieron neoplasias hematológicas (leucemia mieloide aguda y linfoma primario del sistema nervioso central).

Antes del diagnóstico de IDP, el $95 \%$ (n: 38) presentaron infecciones; el 87,5\% (n: 35 ) fueron infecciones recurrentes, y el $63 \%$ (n: 22) de estas afectaron el tracto respiratorio superior y/o inferior. Siete pacientes tuvieron efecto adverso a la vacuna con el bacilo de Calmette-Guérin (BCG) (tres infecciones sistémicas y cuatro locales).

La mediana de edad al inicio de los síntomas fue a los 3,01 meses (mínimo de 00, máximo de 159,83). Al comparar la edad de presentación de síntomas en función del fenotipo de IDP, se observó que las inmunodeficiencias que afectaban la inmunidad celular y humoral (IDCH) fueron

FiguRa 1. Tiempo de retraso en el diagnóstico según el fenotipo de inmunodeficiencia primaria en pacientes pediátricos del Hospital Civil "Dr. Juan I. Menchaca"
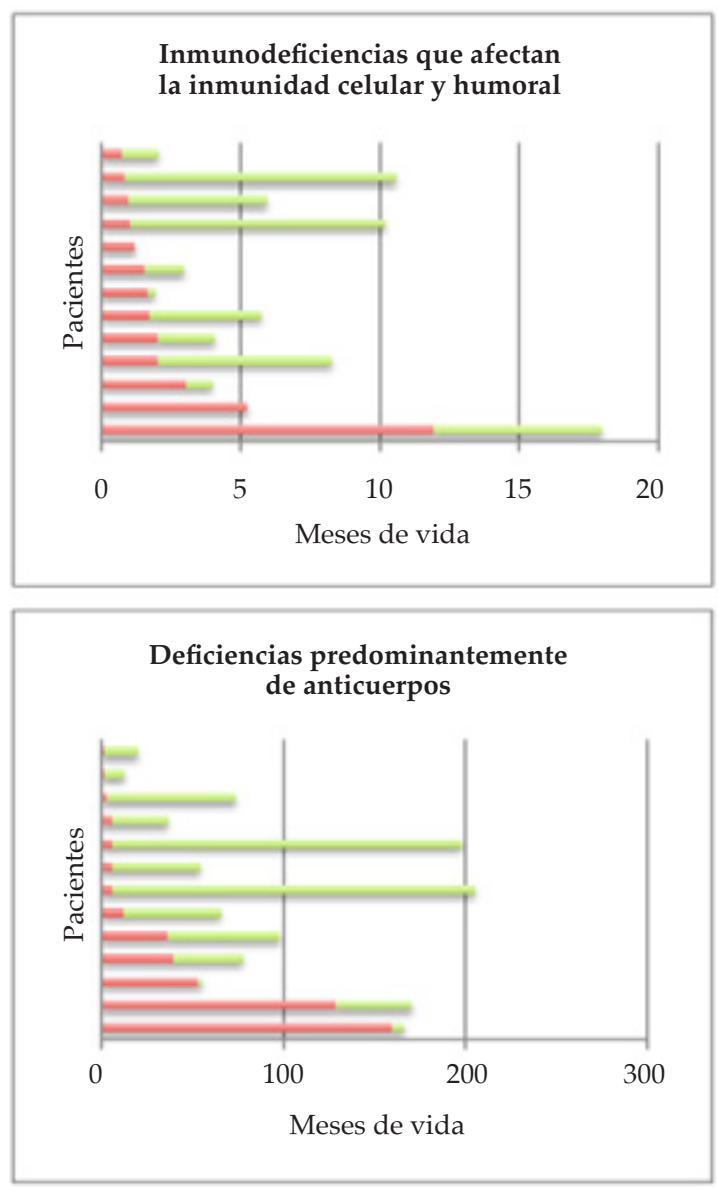

Tiempo de retraso en el diagnóstico de las IDP.

Tiempo de supervivencia posterior al diagnóstico de las IDP.
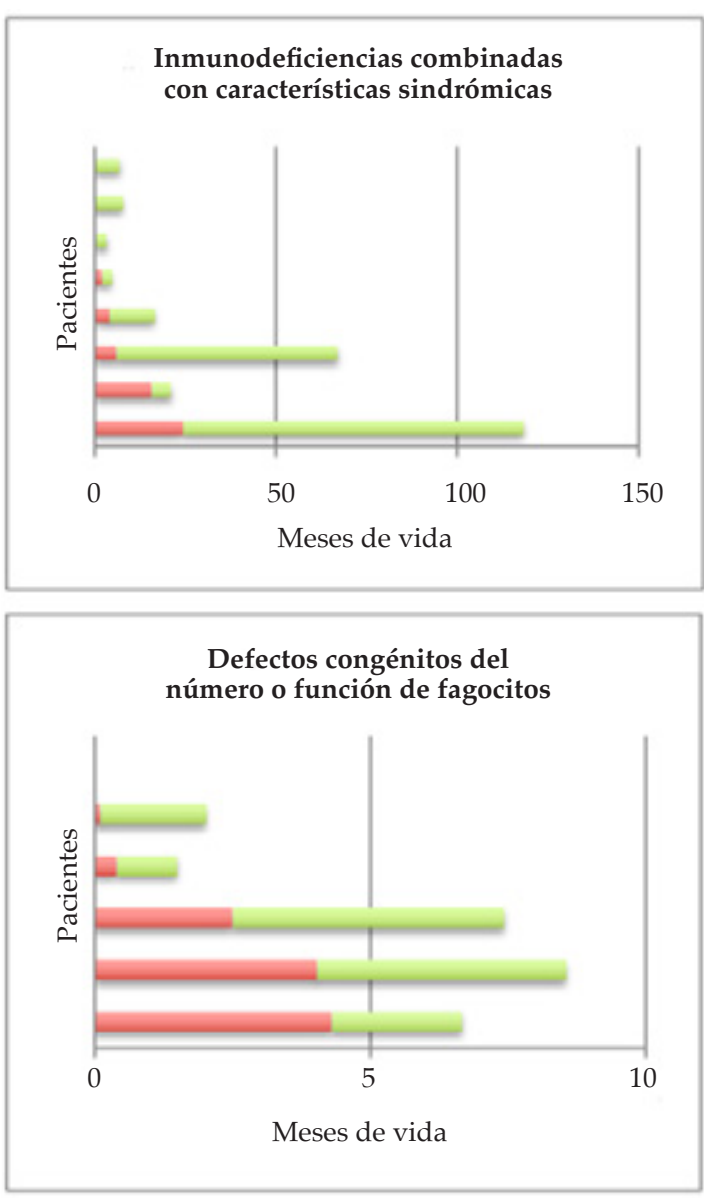
de inicio más temprano (mediana de 1,67 meses, mínimo de 0,73, máximo de 11,9), en comparación con las deficiencias predominantes de anticuerpos (mediana de 6,1 meses, mínimo de 1,57, máximo de 159,83; p <0,001).

La mediana del tiempo de retraso en el diagnóstico fue de 6,3 meses (mínimo de 00, máximo de 199,4). En dos pacientes, se realizó el diagnóstico al momento del inicio de los síntomas por tener familiares de primer grado con IDP. Al comparar el retraso en el diagnóstico según el tipo de IDP, se observó que las deficiencias predominantes de anticuerpos presentaban mayor retraso (mediana de 41,6 meses, mínimo de 2,03, máximo de 199,4) en comparación con las IDCH (mediana de 2,0 meses, mínimo de 0,00, máximo de 9,7; p: 0,008) (Figura 1).

Hasta la fecha del estudio, fallecieron el $35 \%$ (n: 14). Las causas fueron infección sistémica por BCG (3), neumonía (3), sepsis (2), hemorragia cerebral (1), absceso pulmonar (1), absceso abdominal (1), meningoencefalitis (1), infección por adenovirus (1) y enfermedad de injerto contra huésped posterior al trasplante de médula

FIGURA 2. Curvas de supervivencia para pacientes con inmunodeficiencias primarias
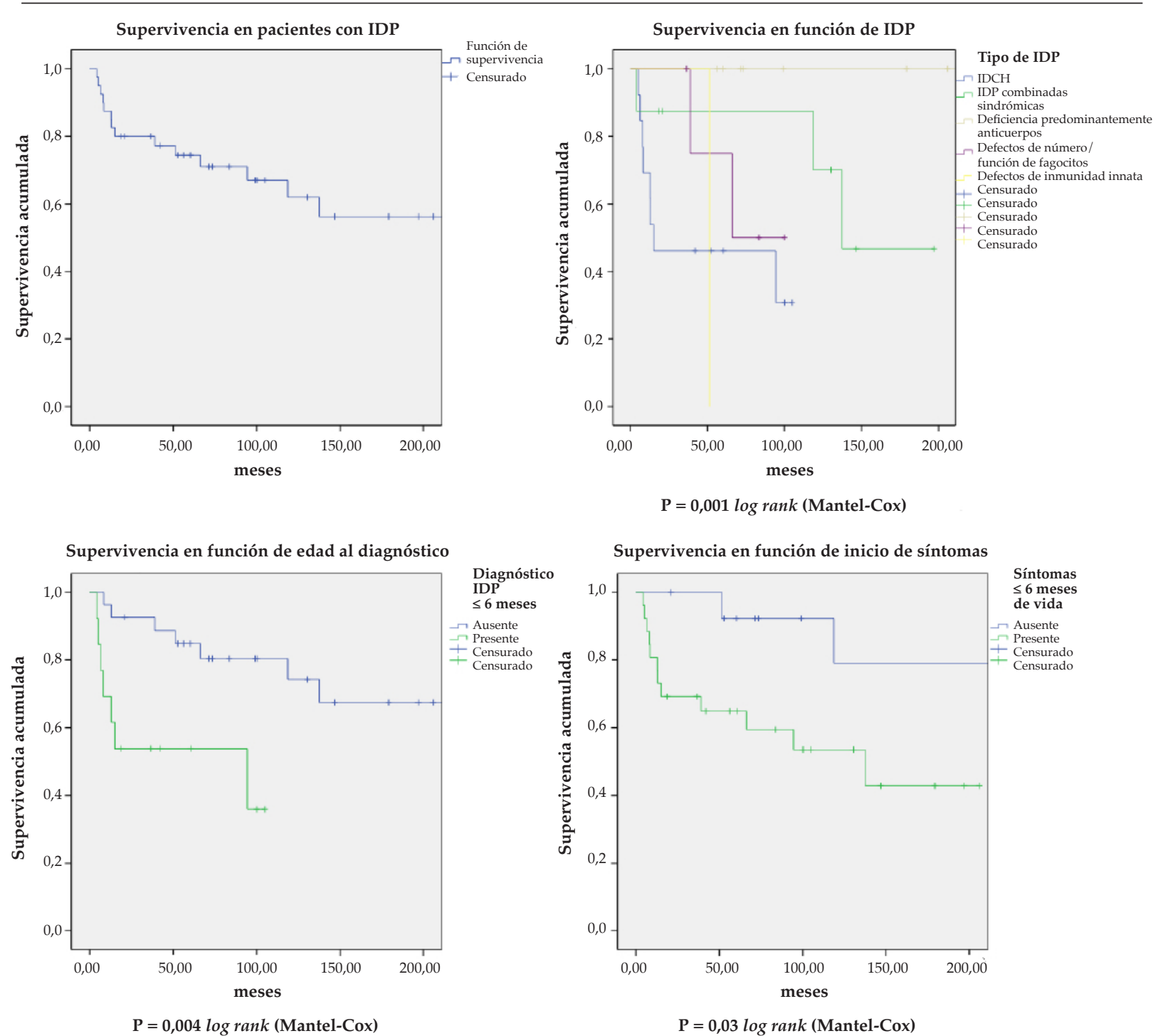

IDP: inmunodeficiencia primaria; IDCH: inmunodeficiencias que afectan la inmunidad celular y humoral. Censurado: pacientes en seguimiento que no fallecieron hasta el momento de realizar este estudio. 
ósea (1). El resto permanecían en tratamiento específico para el fenotipo de la IDP: aplicación de inmunoglobulina intravenosa o subcutánea (13), factor estimulante de colonias de granulocitos (1), antibióticos profilácticos (3), en protocolo para trasplante de médula ósea (1) y en vigilancia posterior a este (8).

En pacientes con IDCH, diez fueron inmunodeficiencia combinada grave (IDCG). De estos, ocho se sometieron a trasplante de médula ósea; cuatro sobrevivieron; un paciente inició el protocolo para terapia génica, pero falleció por una infección por adenovirus. En los niños con síndrome de Wiskott-Aldrich (SWA), cinco recibieron trasplante de médula ósea, de los cuales sobrevivían cuatro.

$\mathrm{Al}$ analizar la mortalidad de los pacientes en función del tipo de inmunodeficiencia, se observó mayor mortalidad en los pacientes con IDCH (el 61,5\% vs. el 22,2 \%, p: 0,038), mientras que, en los pacientes con deficiencia predominante de anticuerpos, no se registró ninguna defunción (el $0 \%$ vs. el $52 \%$, p: 0,001). En la Figura 2, se muestran las curvas de supervivencia.

\section{DISCUSIÓN}

Los pacientes con IDCH y quienes presentaron manifestaciones clínicas o diagnóstico de IDP en los primeros seis meses de vida mostraron menor tasa de supervivencia. Las infecciones fueron la principal causa de muerte.

Similar a nuestros resultados, J. Wu y col., ${ }^{9}$ describieron, en 112 pacientes con IDP, una mortalidad del $38,4 \%$. La edad promedio al inicio de los síntomas y al momento del diagnóstico fue de 13 y 24 meses, respectivamente. Las IDP prevalentes fueron las IDCH (el 28,6\%) y, en pacientes con SWA e IDCG, las manifestaciones ocurrieron a menor edad, 1 y 4 meses, respectivamente.

Mellouli y col., ${ }^{2}$ observaron, en los pacientes con IDP (n: 710), que la edad promedio de inicio de los síntomas fue a los 6 meses y fue menor en las IDCH (1,6 meses) que en las deficiencias predominantemente de anticuerpos (90 meses). Las IDP prevalentes fueron las IDCH (el 28,6\%), y la mortalidad global fue del $34,5 \%$, que fue mayor en las IDCG (el $79 \%$ ).

En los pacientes del HCGJIM con IDP, el tiempo de retraso del diagnóstico fue 6,3 meses y fue mayor en las deficiencias predominantes de anticuerpos. D. Gupta y col., ${ }^{10}$ reportaron, en 120 niños con IDP, un promedio de retraso en el diagnóstico de cinco años. Sin embargo, al comparar este indicador según el tipo de IDP, observaron que, en deficiencias predominantemente de anticuerpos, el retardo fue de 5 años y, en IDCG, fue de 3 meses. Las manifestaciones clínicas tardías en los pacientes con deficiencias de anticuerpos pueden deberse a la transferencia de anticuerpos maternos transplacentarios y a la menor gravedad de las infecciones comparada con la de las IDCH.

Similar a lo descrito en diferentes publicaciones, ${ }^{9-12}$ en los pacientes presentados en esta serie, antes del diagnóstico de IDP, el $87,5 \%$ tuvieron infecciones recurrentes, principalmente, del tracto respiratorio. Otras manifestaciones clínicas de las IDP son los signos y síntomas debidos a autoinmunidad, alergia o malignidad. ${ }^{11,13}$ En el presente estudio, se identificaron tres pacientes con autoinmunidad y dos desarrollaron neoplasias hematológicas. Lugo Reyes y col., ${ }^{11}$ describieron, en niños con IDP, manifestaciones no infecciosas en el $36 \%$ (alergias en el 17\%, autoinmunidad en el 19\%, neoplasias en el 2,4\%).

La historia clínica es una herramienta importante para identificar a pacientes de mayor riesgo de IDP. Los antecedentes familiares justifican, en algunos casos, la búsqueda del diagnóstico antes del inicio de síntomas, y la consanguinidad entre padres incrementa la probabilidad de expresar trastornos autosómicos recesivos..$^{2,10,14} \mathrm{El}$ diagnóstico temprano de las IDP es pertinente. Se ha descrito que el tratamiento oportuno se asocia a mejores resultados en supervivencia y efectos adversos por infecciones. ${ }^{15}$

Las limitaciones de este estudio fueron el acotado número de pacientes y el registro de información retrospectiva. Sin embargo, la información observada permite conocer el comportamiento clínico y la evolución de algunas IDP.

\section{REFERENCIAS}

1. Devonshire AL, Makhija M. Approach to primary immunodeficiency. Allergy Asthma Proc. 2019; v40(6):465-9.

2. Mellouli F, Mustapha IB, Khaled MB, Besbes H, et al. Report of the Tunisian Registry of Primary Immunodeficiencies: 25-Years of Experience (1988-2012). J Clin Immunol. 2015; 35(8):745-53.

3. Benjasupattananan P, Simasathein T, Vichyanond P, Leungwedchakarn $\mathrm{V}$, et al. Clinical characteristics and outcomes of primary immunodeficiencies in Thai children: an 18-year experience from a tertiary care center. J Clin Immunol. 2009; 29(3):357-64.

4. Mahlaoui N, Jais JP, Brosselin P, Mignot C, et al. Prevalence of primary immunodeficiencies in Franceis underestimated. J Allergy Clin Immunol. 2017; 140(6):1731-3.

5. Mendoza-Quispe D, García-Gomero D, Córdova-Calderón 
W. Diagnóstico situacional de las inmunodeficiencias primarias: Aproximación preliminar. Rev Peru Med Exp Salud Pública. 2017; 34(2):346-7.

6. Coria Ramírez E, Espinosa Padilla S, Espinosa Rosales F, Vargas Camaño ME, et al. Panorama epidemiológico de las inmunodeficiencias primarias en México. Rev Alerg Mex. 2010; 57(5):159-63.

7. TangyeSG,Al-Herz W, Bousfiha A, Chatila T, et al. Human InbornErrors of Immunity:2019Update on the Classification from the International Union of Immunological Societies Expert Committee. J Clin Immunol. 2020; 40(1):24-64.

8. Bousfiha A, Jeddane L, Picard C, Ailal F, et al. The 2017 IUIS Phenotypic Classification for Primary Immunodeficiencies. J Clin Immunol. 2018; 38(1):129-43.

9. WuJ, Zhong W, Yin Y, Zhang H. Primary immunodeficiency disease: a retrospective study of 112 Chinese children in a single tertiary care center. BMC Pediatr. 2019; 19(1):410.

10. Gupta D, Thakral D, Kumar P, Kabra S, et al. Primary Immunodeficiency Disorders Among North Indian Children. Indian J Pediatr. 2019; 86(10):885-91.

11. Lugo Reyes SO, Ramírez-Vázquez G, Cruz Hernández A,
Medina-Torres E, et al. Clinical Features, Non-Infectious Manifestations and Survival Analysis of 161 Children with Primary Immunodeficiency in Mexico: A Single Center Experience Over two Decades. JClin Immunol. 2016;36(1):5665.

12. Pedraza Á, Vargas-Rumilla MI, Ramírez-Roa JL. Registro de inmunodeficiencias primariaa en niños en un hospital de cuarto nivel. Bogotá, 2010-2016. Rev Alerg Mex. 2018; 65(4):341-8.

13. Jonkman-Berk BM, Van den Berg JM, Ten Berge IJ, Bredius $R$, et al. Primary immunodeficiencies in The Netherlands: national patient data demonstrate the increased risk of malignancy. Clin Immunol. 2015; 156(2):154-62.

14. BarboucheMR,MekkiN,Ben-AliM,Ben-MustaphaI.Lessons from Genetic Studies of Primary Immunodeficiencies in a Highly Consanguineous Population. Front Immunol. 2017; 8:737.

15. Marciano BE, Huang CY, Joshi G, Rezaei N, et al. BCG vaccination in patients with severe combined immunodeficiency: complications, risks, and vaccination policies. J Allergy Clin Immunol. 2014; 133(4):1134-41. 